\title{
CONCEPCIÓN SOBRE EVALUACIÓN Y PRÁCTICAS EVALUATIVAS DE LOS DOCENTES DEL PROGRAMA DE PEDAGOGI A INFANTIL DE LA UNIVERSIDAD SURCOLOMBIANA
}

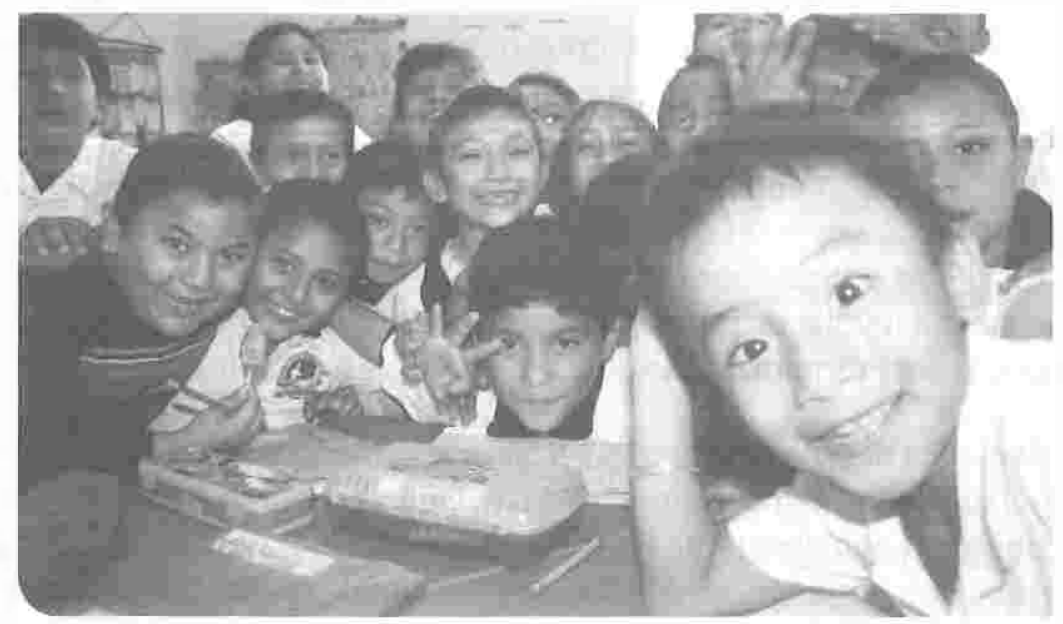

Gloria Mercedes Chavarro Medina ${ }^{48}$

En las últimas décadas, los cambios sociales, económicos, políticos, los avances científicos y tecnológicos, ligados a la globalización, han influido en el proceso educativo, reconociendo un progreso del mismo, lo que ha generado cambios en los paradigmas de la educación, la revisión de enfoques curriculares, la adopción de metodologías y modelos pedagógicos alternos, hechos que hacen necesario revaluar las prácticas y técnicas de evaluación que hasta este momento se han venido realizando.

Es asi como la evaluación educativa, entendida como úna actividad compleja que forma parte de un proceso social mucho más amplio, no puede ser considerada un proceso autárquico, independiente, aislado, atomizado; por el contrario, hace parte del proceso de formación, al igual que las estructuras curriculares y las prácticas pedagógicas ${ }^{49}$; por esta razón ha suscitado discúsiones, debates y

49 Grupo de finvestigación PACA. Estado del Arte: Evaluación de la Caliodad de b Educación en Colombia. 
reflexiones en los últimos años desde los entornos educativos; debido a esta preocupación han aumentado de forma significativa el número de investigaciones sobre la innovación que demanda la dinámica de la evaluación de los aprendizajes.

\section{El porqué del proyecto}

En el Programa de pedagogía infantil de la Universidad Surcolombiana se puede evidenciar una problemática latente, que preocupa a docente y directivos y es sobre las prácticas profesionales que se vienen desarrollando, se ha podido observar en estas prácticas que la calidad del desempeño de las estudiantes no corresponde con el nivel de los puntajes obtenidos en las evaluaciones realizadas por los docentes dentro de las prácticas evaluativas del Programa. Estas situaciones podrían generarse por brechas o tensiones entre lo planeado y lo ejecutado e involucra, por derivación, a la comunidad educativa, es decir, a las directivas del Programa, a los docentes y a los estudiantes.

La problemática descrita se agudiza en las prácticas evaluativas finales que tiene estipulado el Programa, cuando el desempeño esperado de los estudiantes. no es coherente con el sistema de valoraciones o resultados de la evaluación cuantitativa qúe obtienen en sus diferentes asignaturas, lo cual deja tanto falencias en la articulación entre la evaluación del proceso y la evaluación final, como incongruencias en relación con los propósitos del Programa, según se deriva de los registros e informes de los asesores de práctica y en las qúejas que instauran los estudiantes en sus consejerías.

Por otro lado, cabe resaltar que para la planeación cúrricular, el Consejo Superior de la USCO ha definido una estructúra formal académica qúe le permite al docente contar con una herramienta orientadora en los términos del Acuerdo 048 del 2005, que en el artículo segundo plantea: "Todo curso debe tener un Microdiseño Curricular estructurado por semanas..."; allí se establece y ad junta el formato oficial del microdiseño curricular, que si bien incluye criterios de evaluación no precisa de qué manera se están llevando éstos a la práctica y qué uso se les da a los resultados de la evaluación y, mejor aún, que incidencia tiene tal encuadre normativo en la praxis educativa, entendida como la articulación entre la teoría y la práctica que debe conducir a los estudiantes por vías o caminos para alcanzar los objetivos de formación

De lo anterior surgió la necesidad de reflexionar sobre ¿Cuáles son las concepciones sobre evaluación del aprendizaje por parte de los docentes del Programa de Pedagogia infantil de la Universidad Surcolombiana, y cómo se articulan con sus practicas evaluativas, en el año 2011 ? 


\section{El derrotero metodológico}

La investigación es de tipo cualitativo-cuantitativo (mixto), en los términos referidos por Hernández Sampieri y otros, cuando afirman: "La meta de la investigación mixta no es remplazar a la investigación cuantitativa ni a la investigación cualitativa, sino utilizar las fortalezas de ambos tipos de indagación combinándolas y tratando de minimizar sus debilidades potenciales ${ }^{50 "}$.

Asi mismo, tuvo un enfoque descriptivo - propositivo dado que se pretendió describir y analizar las concepciones que tienen los docentes y estudiantes del Programa de Pedagogía Infantil de la Universidad Surcolombiana en el 2011 frente a la evaluación e identificar las prácticas evaluativas desarrolladas por los docentes y a la luz de los resultados se diseñó una propuesta evaluativa inmersa en el enfoque pedagógico y los propósitos de formación del Programa que permita a los estudiantes ser partícipes del proceso de evaluación.

\section{Las audiencias}

Estuvo conformada por los 8 docentes de tiempo completo pertenecientes al Programa de Pedagogía Infantil de la Universidad Surcolombiana, seleccionados por el criterio de mayor dedicación al Programa, dado el tipo de vinculación y 12 docentes catedráticos que manifestaron su interés y disposición para participar en la entrevista. La muestra de estudiantes se definió, arbitrariamente, en un $10 \%$ de la población, dada la modalidad del cuestionario para indagar en profundidad, algunos de los aspectos de la evaluación. Estos 35 estudiantes se seleccionaron de manera aleatoria.

\section{Conclusiones}

Las conclusiones obtenidas en el marco de este proyecto fueran las siguientes:

En los documentos institucionales no está explicito un soporte o planteamiento teórico básico que le de identidad al componente evaluativo del Programa; es así como al indagar sobre el proceso de evaluación se evidenció queen los microdiseños no existen parámetros que lo definan; sobre los documentos PEP, PEU y el Manual de Convivencia, se puede afirmar que la evaluación es abordada sin un eje articulador y presenta una heterogeneidad de criterios para la tipología de la evaluación como producto de la multiplicidad de concepciones alifi presentes.

50. HERNANDEZ, R, FERNANDEZ, C, BAPTISTA, L_(2006), Metodologfa de le irwestigación, México: McGraw Hill-Interamericana. 
En los microdiseños, por ejemplo, nose explicitan los criterios y tipos de evaluación en los cuales los docentes podrían encontrar efementos teóricos conceptuales comunes para articular el proceso evaluativo en sus asignaturas.

En torno a las estrategias de evaluación propuestas en los documentos, se observa que predomina la evaluación escrita, donde se privilegia la verificación de contenidos adquiridos por el es tudiante para emitir una calificación, propia de un modelo tradicional que corrobora resultados de jando de lado el carácter permanente y sistemático de la evaluación y restando impor tancia a la autoevaluación, heteroevaluación y coevaluación, las cuales nose hacen visibles en los microdiseños curriculares.

En consecuencia, los resultados encontrados a partir de las respuestas de los docentes y estudiantes, el panorama revelado es producto de la indefinición teórica, metodológica y técnica de la USCO y no de la falta de criterios de cada docente. En otras palabras, en el campo de la evaluación del aprendizaje, la institución tiene una tarea por hacer y debe ser hecha de manera prioritaria.

\section{Propuesta}

La Evaluación Integral: Una Alternativa Hacia La Calidad. Propuesta Evaluativa Para El Programa De Pedagogía Infantil

Es importante resaltar la distinción existente entre la evaluación sumativa y la formativa; en la primera, se busca definir una nota centrada en el producto o resultado que permita la aprobación o no del curso; en la evaluación formativa, se busca dar cuenta del proceso y desarrollo intelectual del alumno. En la actualidad ha surgido otro concepto: la evaluación formadora, sustentada por autores como Bordas y Cabrera, quienes afirman:

"Si la evaluación formativa es una respuesta a la iniciativa docente, centrada en la intervención del profesor, tanto en la información facilitada como en la recogida de información, la evaluación formadora arranca del propio discente; esto es, se fundamenta en el autoaprendizaje; la evaluación formativa es una respuesta a la iniciativa docente, mientras que la evaluación formadora responde a la iniciativa del discente ${ }^{\prime 51}$.

Al surgir del alumno la necesidad de aprendizaje, al partir de esa autoevaluación y reflexión propia de sus conocimientos, el estudiante estará motivado y será un participante activo durante el desarrollo del proceso

51 BORDAS, Inmaculada y CABRERA, Flor (2001 ) Estrategia de Evaluación de los Aprendizajes Centrados en el Proceso. En Revista Espan̄ola de Pedagogía. Edición Enero- abril. NP 248.Pág 31. Barcelona. 
educativo; él mismo generará preguntas y de él mismo nace la necesidad de dar respuesta a ellas.

En el Proyecto Educativo del Programa de Pedagogía Infantil de la Universidad Surcolombiana, se observa que, si bien en su contenido se mencionan elementos para la orientación del proceso evaluativo de los docentes hacia una evaluación centrada en el proceso, tal como se mencionó anteriormente, así como los tipos de evaluación del Programa (Autoevaluación, Coevaluación y Heteroevaluación), la adopción del modelo Constructivista Social como enfoque pedagógico y los criterios de evaluación, es evidente la carencia en la profundización conceptual de los mismos. En la búsqueda del mejoramiento continuo, la presente propuesta hace énfasis en fortalecer los lineamientos y orientaciones estipulados en el Programa, asi como las prácticas evaluativas de los docentes, en dos grandes dimensiones: fundamentación teórica conceptual y ejes temáticos.

Fundamentación Teórica-Conceptual. Desde una perspectiva tradicional, la evaluación es una herramienta de control en la cual el docente puede identificar lo que el alumno entiende y hace a partir del conocimiento impartido, mientras que la evaluación vista desde los procesos de aprendizajes es una estrategia de formación permanente, tal como lo afirman Bordas y Cabrera:

"La evaluación, incluida en el mismo acto de aprendizaje, comporta una mayor comprensión tanto por parte del profesor como del estudiante de los procesos gue se están realizando así como el conocimiento de las razones de los errores y aciertos que se producen. El acto evaluativo, desde esta perspectiva, másque un proceso para certificar o aprobar, se coloca como participante, como optimizador de los aprendizajes contribuyendo a proporcionar información relevante para introducir cambios y modificaciones para hacer mejor lo que se está haciendo"52.

En el Proyecto Pedagógico del Programa de Pedagogía Infantil como ya seespecificó en el desarrollo de esta investigación, se retoman estos criterios del modelo constructivista y, desde esta óptica, se hace necesario que el estudiante sea capaz de evaluarse de una forma objetiva y válida, que reconozca la capacidad de aprendizaje de las diferentes situaciones, que promueva la apropiación de los objetivos de su aprendizaje, así como de los criterios de evaluación.

Se busca una construcción del aprendizaje a partir del consenso entre el alumno y el docente, la creación o selección conjunta de los criterios de evaluación, de las actividades a evaluar y los estándares de logros. Se invita a los docentes 
a construir sus estrategias evaluativas basadas en situaciones lo más parecidas posible a la realidad, que le permitan al alumno plasmar de una forma práctica sus conocimientos. Se hace entonces factible la incorporación de estos elementos evaluativos a los microdiseños curriculares de las asignaturas, que le permitan al alumno tomar conciencia de los conocimientos que ha adquirido hasta el momento y las dificultades que se presentan en su aprendizaje, para que el estudiante y el docente identifiquen cual es la mejor estrategia para superar esas falencias.

Al respecto, las autoras antes citadas que sustentan este tipo de enfoque plantean que "Las estrategias de evaluación de naturaleza metacognitiva tales como los diarios reflexivos, el portafolio, la autorregulación del aprendizaje mediante la elaboración de mapas conceptuales, la auto observación y valoración de las adquisiciones mediante el uso de plantillas de evaluación son recursos favorecedores de una evaluación centrada en el proceso másque en las resultados"s53.

Así, desde éste enfoque, la evaluación como herramienta, propicia en el alumno la capacidad de aprender a evaluar su propio aprendizaje, aspecto esencial al momento de aprender a aprender.

Las estrategias de evaluación centradas en el proceso de aprendizaje son muchas y se pueden utilizar como herramienta de evaluación de los aprendizajes centrados en el proceso. Entre ellas, surgen dos que podrían de una forma didáctica y reflexiva evaluar continuamente el aprendizaje de los estudiantes del Programa de Pedagogía Infantil de la Universidad Surcolombiana:

El Portafolio, entendido como "una colección selectiva deliberada y variada de las trabajos del estudiante donde se reflejan sus esfuerzos, progresos y logros en un periodo de tiempo yen alguna área específica"s4.

Desde esta herramienta de evaluación se promueven aprendizajes positivos a partir de situaciones problemas y la identificación por parte del estudiante de estrategias para la ejecución de las tareas. Este se compone de materiales de trabajos obligatorios y opcionales, concertados entre el docente y el estudiante y le permite a este último profundizar libremente en cada una de las temáticas obligatorias.

Algunos de los tipos de portafolios que se consideran de mayor relevancia y facilidad de ejecución para el caso del Programa son: 
Portafolio de trabajo: en éste el estudiante y el docente realizan una revisión diaria de los temas estudiados y las actividades realizadas por el alumno, comprobando el progreso en el aprendizaje.

Portafolio de presentación: en este tipo de portafolio, el estudiante presenta en las fechas establecidas una selección de los que a su pensar son los mejores trabajos. Dentro de esta presentación se pueden incluir los trabajos de progreso y los trabajos diarios.

Portafolio de recuerdo: se construye a partir de todos los materiales que no fueron incluidos en el portafolio de presentación y a través de los cuales se puede evidenciar el progreso en el aprendizaje.

Otra estrategía que se considera pertinente en esta propuesta son los Mapas Conceptuales, que permiten identificar la apropiación que realiza el estudiante de los nuevos conocimientos, la articulación mental de los conceptos; su uso continuo y sistemático, hace posible identificar a tiempo falencias o dificultades en la comprensión de los conceptos por parte del mís mo alumno.

Para autores como Novack y Gowin, citados por Bordas y Cabrera, "Ios mapas conceptuales son diagramas que expresan las relaciones entre conceptos generales yespecificos de una materia reflejando la organización jerárquica entre ellos. Es una técnica que se utiliza tanto en la enseñanza como en la evaluación y favorece al desarrollo organizado y funcional de los conceptos claves de una materia o disciplina"ss.

Se debe destacar que cualquier herramienta evaluativa adquiere el sentido que el docente o alumno k atribuyan siempre y cuando se explicite su soporte teórico-conceptual. Luego se pueden operacionalizar de distintas formas, según la actitud con la que se aborda el proceso evaluativo, puesto que "algumos términos como diálogo, consenso, flexibilidad, autorreflexión, coevaluación y participación deben animar la actividad evaluativa si se pretende que tenga un impacto en la calidad de los procesos de aprendizaje y si queremos que el estudiante aprenda a evaluar ${ }^{\prime \prime 5 \sigma}$.

Es de advertir que desde esta propuesta evaluativa no se está descalificando la utilización de estrategias evaluativas tradicionales, (evaluaciones escritas con fines cuantitativos, exposiciones que lleven el aprendizaje memorístico, entre otros), sólo se recomienda que su adopción responda y se corresponda con el enfoque y concepción evaluativa que se haya explicitado sobre las mismas, así como la inclusión de los ejes temáticos dentro del proceso evaluativo realizado por los docentes. 


\section{Ejes Temáticos}

\section{Articulación de los ejes temáticos.}

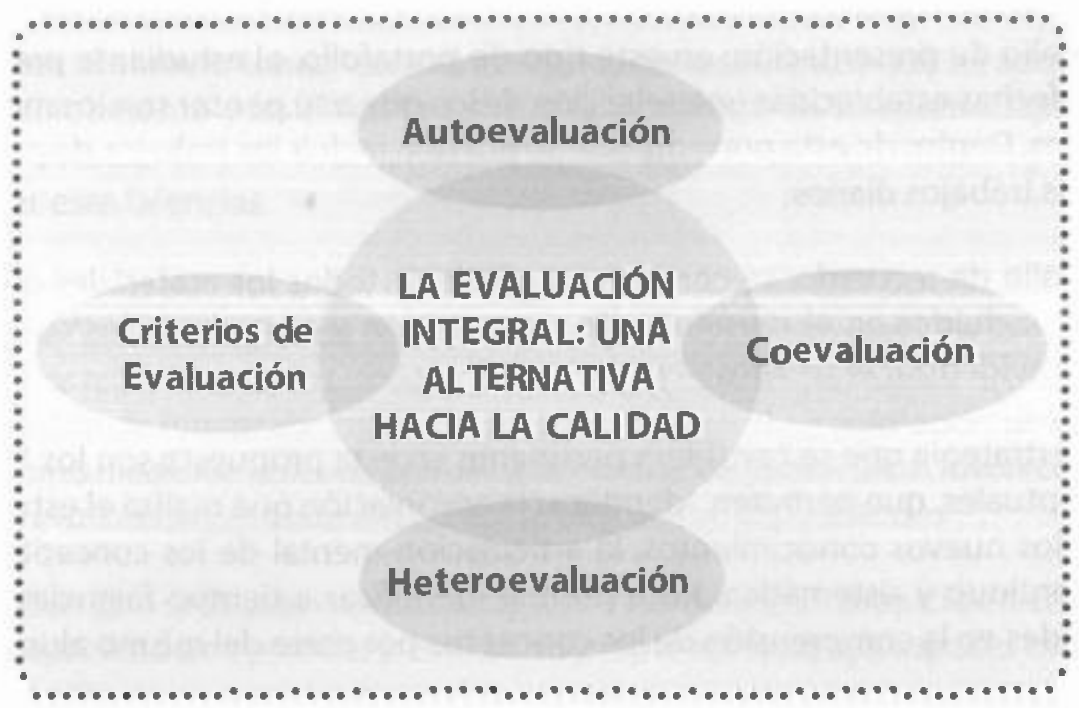

Primer eje temático: Autoevaluación. La autoevaluación debe ser entendida como un proceso de reflexión en donde el alumno tome conciencia de sí mismo, de sus objetivos y logros, y el docente será el guía que oriente y acompañe al estudiante hacia el logro de estos objetivos

Como afirma Herrera: "La autoevaluación es una forma de evaluación compartida entre el profesor y el alumno, donde el esfuerzo concertado entre las partes, se erige como garante de la más clara ob jetividad"s.

En la medida que el estudiante aprende a evaluarse también aprendie a identificar sus necesidades, sus fortalezas y a establecer expectativas y metas, a partir de la autoevaluación el alumno puede formular planes de acción para conseguir sus logros y valorar los avances en su proceso de aprendizaje.

En este sentido, Bordas y Cabrera han afirmađo que"La metacognición es aquella habilidad de la persona que le permite tomar conciencia de su propio proceso de pensamiento, examinarlo y contrastarlo con el de otros, realizar autoevaluaciones y autorregulaciones. Es un "diálogo interno" que nos induce a reflexionar sobre to qué hacemos, cómo lo hacemos, y por qué lo hacemos".

57 HERRERA, Marta (2001). La autoevaltwación en las prácticas pedagógicas de los alumnos del profesorado en enseñanza primaria. Facultad de Ciencias de la Educación. Universidad Nacional de Conahue, Argentina. En: Revista lberaamericana de Educación. 
La autoevaluación que realizan los estudiantes, no es solo un requisito para la aprobación de un curso, sino que también puede convertirse en una herramienta para la redefinición de la función docente. Es así como a partir de la realización de la autoevaluación, se propicia un espacio de diálogo entre el docente y el estudiante, que se constituye en una herramienta para la obtención de información sobre el proceso evaluativo.

Para la puesta en marcha de la Autoevaluación se cuenta con diferentes tipos de estrategias, de las cuales se sustentan dos, consideradas de vital importancia:

La Autoevaluación a partir del enfoque de "Cuestionario de Autoevaluación". En este tipo de enfoque se propone la utilización de cuestionarios, a partir de la creación de criterios de evaluación y el establecimiento de rangos de respuesta, ya sea: SI, NO ode 1 a 5.

Otra estrategia seria el enfoque de Autoevaluación por "Reunión de trabajo". En esta se puede evidenciar una implicación más activa del docente en el proceso, en un primer momento se realizará una autoevaluación de tipo cuestionario y luego una socialización en la cual el docente busca un consenso final sobre la nota.

Segundo eje temático Coevaluación: La coevaluación puede entenderse como la percepción sobre el trabajo colaborativo que desempeñan el docente y sus alumnos.

Algunos autores, como Gessa ${ }^{58}$, la consideran parte de un conjunto de procedimientos denominados "Evaluaciones Alternativas", el cual difiere de las estrategias evaluativas de medición deł aprendizaje a partir de pruebas objetivas e incursiona en el proceso evaluativo la evaluación a partir de tareas reales.

Se puede definir la Coevaluación como la evaluación conjunta realizada por el docente y los estudiantes, quienes llegan a un consenso en la valoración o calificación; la coevaluación le permite al estudiante identific ar sus falencias o dificultades a partir del reconocimiento de las mismas por parte de sus compan̄eros y docentes, dando a esta mayor imparcialidad queen la autoevaluación. La calific ación del estudiante habrá de surgir de una ponderación sobre la nota identificada por el docente y las apreciaciones realizadas por los estudiantes, esta ponderación sumară a la nota final aprobatoria del estudiante, con un porcentaje previamente establecido por el docente.

58. GESSA PERERA, Ara (200)). La coevaluaçión como metodologia complementaria de b evaluaclón del aprendizaje. Anălisis y reflexión en las aulas universitarias. Enc Revista de Educación, N0 354. Enero-Abrit. En http//wwwwrevistaeducacion.mec.es/re354/re354_30.pdf. Consultado el 02 de julio de 2012. 
El desarrollo de la evaluación es inherente a ambientes de aprendizaje, en los cuales se reconoce al alumno como el elemento central en su proceso educativo. Este tipo de evaluación puede ser llevado fácilmente a la práctica por los docentes del Programa de Pedagogía Infantil de la Universidad Surcolombiana.

Una estrategia para la implementación de la coevaluación en las asignaturas del Programa podría ser:

Se realiza la asignación de trabajos grupales a los estudiantes sobre diferentes temáticas de la asignatura, transcurrido el plazo para la elaboración de los trabajos, se hace la socialización ante docente y alumnos para su evaluación conjunta. Posterior a la socialización de cada uno de los trabajos, los estudiantes y el profesor evaluaran individualmente todos los grupos, a excepción del suyo, en una escala de puntuación previamente definida. Entre los criterios de evaluación que podrían incluirse se sugieren: relaciones entre conceptos, creatividad y originalidad en el aborda je del tema, capacidad de síntesis, coordinación, entre otros.

Esta estrategia hace viable la coevaluación que se da entre pares (alumnos), y permite identificar y comunicar habilidades y destrezas percibidas por los compañeros.

Tercer eje temático: Heteroevaluación. La heteroevaluación, desde lo conceptual y lo metodológico, se constituye en una herramienta que tiene lugar a partir de la evaluación de una persona a otra, pero de diferente nivel, basándose en los procesos, capacidades, habilidades, actitudes y rendimiento.

Si se considera como una herramienta evaluativa desde los estudiantes, entonces, seria entendida como, la evaluación que el alumno realíza sobre el desempeño académico del docente durante el transcurso del curso y podría hacer las sugerencias que considere convenientes en tanto estén fundamentadas.

La Secretaria de Educación Pública de México, considera que "La heteroevaluación es la medición que el profesor hace del logro de las competencias en sus alumnos, mediante la interacción profesor-alumno se obtienen consensos sobre los factores que influyen en torno de los aprendizajes esperados en los estudiantes" ${ }^{\prime \prime}$.

59 SECRETARIA DE EDUCACIÓN PÜBLICA. La Evaluación en la Enseñanza y el Aprendizaje. Geografia de México y dél mundo. [En línea]. Información disponible en httpy/www.reformasecundaria,sepgob.mx/geografia/doc/evatuacion.pdf. Consultado eli of de julio de 2012. 
Desde la heteroevaluación el docente puede cuestionarse si las estrategias y herramientas utilizadas fueron las correctas y condujeron finalmente al aprendizaje de los estudiantes. Además, le permite reflexionar sobre las competencias adquiridas por el alumnado, cuáles sus fortalezas y cuáles sus debilidades.

La puesta en marcha de la heteroevaluación, no requiere de la elaboración compleja de instrumentos de evaluación, esta puede ser implementada por los docentes hacia sus estudiantes, por medio de la creación desde la directivas del Programa de un cuestionario corto, dirigido al profesor en el cual él pueda reflexionar sobre su desempeño y contribución al proceso educativo del alumnado y para la heteroevaluación desde los estudiantes igualmente con un cuestionario estandarizado que permita evaluar el desempeño académico del docente.

Cuarto eje temático: Criterios de evaluación. La planeación de un proyecto ode un proceso de evaluación demanda especificar cuáles y cuấntos son los criterios que se definirán como elementos de contrastación de procesos y de resultados. Sobre el particular, Ruiz Córdoba ha expuesto:"

Los criterios de evaluación son los referentes inmediatos de la evaluación porque traducen las capacidades al lenguaje de comportamientos y conductas directamente observables y medibles" (...) "Por ello los criterios de evaluación concretan, de manera clara y precisa, los tipos y grados de conductas y acciones relacionadas con el aprendizaje que debe realizar el alumnado, a propósito de los bloques de contenido previamente seleccionado $\%$.

Sobre este mismo eje, Bosque señala que los criterios de evaluación deben responder a la pregunta sobre quể evaluar y afirma que los criterios "son indicadores sobre qué aprendizajes son los que debe conseguir el alumno al finalizar la tematica. Por lo tanto, hacen referencia a la consecución de las metas propuestas al principio de la Unidad, es decir, los objetivos didácticos" ${ }^{26 ?}$.

Es claro, al revisar la bibliografía sobre el tema, que los criterios se basan en los objetivos del curso o del programa ya partir de éstos se pueden interpretar los resultados con respecto a los objetivos.

Ya se han mencionado las estrategias metacognitivas y su papel en la auto evaluación y coevaluación, pero para que estas se puedan realizar correctamente,

60 Rill2 C Marla del Carmen (201 0) La ampiliación der concepto de evaluación. En: Revis ta Digitai innovación y experiencias educativas, \$27, Febrerode 2010. Andalucla, España.

6: BOSQUE B, Rut. Orientaciones para realizar Unidades Didácticas. En: http//www.reformasecundarla.sep.gob.mx/geografia/doc/evaluacion.phttp:/www.pr of es.net/rep_documentos/Monograf/4GDOpo05Evaluacion.pdf. Consultado: 02 dejuliode2012. 
el alumno requiere conocer los criterios e indicadores de evaluación que se han de tener en cuenta para valorar sus acciones, procedimientos y productos.

Desde la perspectiva de la evaluación del aprendizaje centrada en el proceso, se hace necesario que estos criterios se construyan conjuntamente por el docente y el estudiante, propiciando un aprendizaje autentico y significativo la estrategia de aplicación de este eje temático, demanda la elaboración consensuada entre estudiantes y el docente de los criterios de evaluación.

Es de anotar que desde el Proyecto Educativo del Programa de Pedagogía Infantil, se estipulan unos criterios de evaluación, enunciados a continuación, de los cuales se pueden seleccionar e incorporar en los microdiseños curriculares los que se consideren tenga mayor pertinencia.

\section{Criterios de Evaluación señalados en el Proyecto Educativo del Programa de Pedagogía Infantil PEP- Universidad Surcolombiana}

- Las actividades escolares o de aula (teórica y práctica)

- Las actividades adicionales preparatorias y consecuente estudio previo del material y el cumplimiento de las actividades de aprendizaje.

Elaboración de trabajo de síntesis individual y colectiva

Investigación de ensayos

\section{Operacionalización De La Propuesta}

Para la puesta en marcha de esta propuesta, se hace necesario en un primer momento la socialización del Proyecto Educativo del Programa, del Proyecto Educativo Universitario y del Modelo Pedagógico del Programa.

A partir de aquí, se debe realizar una socialización de la propuesta con todos los docentes del Programa, para la implementación de las diferentes estrategias expuestas en cada una de sus asignaturas, así como la construcción de otras estrategias que se adapten a las necesidades del Programa de Pedagogía Inf antil de la Universidad Surcolombiana. 
A su vez, para identificar la pertinencia y efectividad de la propuesta, se propone la implementación inicial de la misma en cuatro asignaturas del Programa, que servirían como una especie de pilotaje, y realizar una comparación al final del semestre con las otras asignaturas, en materia de percepción tanto del alumno como del docente de su proceso evaluativo, facilidad de implementación de la propuesta y acogida de la misma.

\section{Evaluación De La Propuesta}

La evaluación de esta propuesta, incorporará elementos de análisis derivados de:

- La retroalimentación que se realice en cada asignatura al finalizar el semestre.

- La evaluación que los alumnos realizan de su proceso evaluativo

- La pertinencia y facilidad de implementación que los docentes identifican de las diferentes estrategias planteadas

- La valoración que se realice sobre la contribución o no de esta propuesta al logro de los objetivo de formación del Programa.

A partir de este anălisis se revisará, reformulará y se reestructurará la propuesta, para ajustarla a las necesidades propias del Programa. Es de esperar que forme parte también de la autoevaluación institucional y como ya se mencionó esté presente en el plan de mejora del Programa de Pedagogía Inf antil.

\section{Referente Bibliógrafico}

AHUMADA, Pedro. (2001). La Evaluación en una Concepción de Aprendizaje Significativo. Universidad Católica de Valparaíso: Ediciones Universitarias de Valparaíso.

ALFARO de MALDONADO, M. (2000). Concepción constructivista de la evaluación. Evaluación del Aprendizaje. Caracas: Fondo Editorial de la Universidad Pedagógica Experimental Libertador, FEDUPEL.

\section{ALTAREJOS y Cols. (1998). Ética docente. Barcelona: Ariel.}

BECERRA-BULLA, Fabiola (2008). Concepción y tendencias en la evaluación del profesor universitario: el caso de la Universidad Nacional de Colombia, Facultad de Medicina, Departamento de Nutrición y Terapias, primer semestre de 2005. En: Revista de la Facultad de Medicina.2008; 56. 
CABRA, Fabiola (2007). La evaluación de los aprendizajes en la educación superior. Apuntes críticos para un concepto integrador. Formas de educación No. 8. Bogotá: Javegraf.

CAJAO RESTREPO, Francisco. Pedagogía de las Ciencias Sociales. Fundación FES. Bogotá: Tercer Mundo Editores.

CARDINET, Jean (198ó). Evaluación escolar y medición.

CATAÑO, Gonzalo (2001). Educación y sociología. México: Ediciones Coyoacán. CONGRESO DE LA REPUBLICA (1992). Ley 115 (Ley General de Educación).

DE ZUBIRíA SAMPER, Julián (200ó). Los modelos pedagógicos: Hacia una pedagogía dialogante. Bogotá: Cooperativa Editorial magisterio.

DE ZUBIRIA, Miguel. y DE ZUBIRIA, Julián (1986). Fundamentos de Pedagogía Conceptual. Una propuesta curricular para la enseñanza de las ciencias sociales para pensar. Bogotá: Presencia.

DIARIO OFICIAL. Constitución Política de Colombia. 1991.

DIAZ, Frida,; BARRIGA, Arceo y HERNÁNDEZ, Gerardo (2006). Estrategias docentes para un aprendizaje significativo. Una interpretación constructivista. México: Mc Graw Hill 2006.

DIAZ, Mario (2008). Sobre el currículo: más allá del concepto. Introducción a una semiótica del currículo. En: Revista Colombiana de Educación Superior.

FERREIRO, Ramón. Más allá de la teoría: el Aprendizaje Cooperativo y el Constructivismo Social. Nova SoutheasternUniversity. (s. c.)

FLÓREZ, Rafael (1993). Constructivismo pedagógico y enseñanza por procesos. Bogotá: McGraw Hill.

GLAZMAN N., Raquel (2001). Evaluación y exclusión en la enseñanza univerșitaria. Barcelona: Paidós.

GÓMEZ, Miguel Angel (2001). Pedagogía: Definición, métodos y modelos. En: Revista Ciencias Humanas. №. 2ó. Universidad Tecnológica de Pereira.

GONZÁLEZ, María y HERNANDEZ, Ana (2006). El constructivismo en la evaluación de los aprendizajes del álgebra lineal. Universidad del Zulia, Venezuela. 
HAD J, CH. (1992). Los enfoques actuales de la evaluación y sus implicancias en la práctica en el aula."Paris: ESF.

LUJAN B. María (2008). Prácticas de evaluación dominantes en los alumnos del nivel universitario de enseñanza. Biblioteca y Centro de documentación. Hemeroteca digital. Universidad Nacional Tres de febrero.

KANT, Immanuel (1985). Tratado de Pedagogía. Trad. Colegio Mayor de Nuestra Señora del Rosario; Bogotá: Ediciones Rosaristas.

IAFRANCESCO, Giovanni (2004). La evaluación integral y del aprendizaje. Bogotá: Editorial Magisterio.

LOPEZ, Nelson (2001). La de-construcción curricular. Bogotá: Editorial Magisterio.

LOPEZ SILVA, Saúl y otros (2004). Contenidos Esenciales (SYLLABUS) para la educación de postgrado en ciencias de laboratorio clínico. En: Revista Bioquímica. Octubre-Diciembre. Volumen 29, Numero 004. México.

LUKAS y SANTIAGO (2004). Evaluación de centros escolares. En: Revista Electrónica de investigación Educativa. Volumen 6, № 2.

MAGEDZO, Abraham (1986). Currículum y Cultura en América Latina. En: www. educacion.usach.cl/educacion/files/file/.../SOClOLOGIA.pdf.

MENIN, Ovide (2001). Pedagogía y Universidad. Currículo, Didáctica y Evaluación. Bogotá: Editorial Homo Sapiens.

MOLINA BEDOYA, Víctor A. y otros (2009). La práctica formativa en el campo de la motricidad en contextos de realidad. En: Pedagogía, educabilidad y formación de docentes. Zambrano Leal, Armando. Cali; Artes Graficas del Valle.

MORENO OLIVOS, Tiburcio (2009). La evaluación del aprendizaje en la universjdad. Tensiones, contradicciones y desafíos. En; Revista Mexicana de Investigación Educativa, Vol. 14, Núm. 41, abril-junio.

NASSIFF, Ricardo. Teoría de la educación. Problemática pedagógica contemporánea. Editorial Cincel.

NIRENBERG, Olga (2012). Nuevos enfoques en la evaluación de proyectos y programas sociales, información:En:

http://cdi.mecon.gov.ar/biblio/docelec/clad/cong6/7nov/49/nirenber.pdf, información consultada el 20 de Junio de 2012. 
NIRENBERG, Olga y otros (2003). Programación y Evaluación de Proyectos sociales. Aportes Para la Racionalidad y la Trasparencia. Buenos Aires: Paidós.

OSORIO, Luis Gilberto. Currículo concreto pensado de la constitución actual de los sujetos. Informe final de la investigación realizada para optar al título de Especialista en Gestión de Procesos Curriculares de la Fundación Universitaria Luis Amigó (s. c.).

PINILLA, Analida y SANCHEZ, Hugo (2001). Diagnóstico sobre la evaluación açadémica en pregrado realizada por los docentes de la Facultad de Medicina de la Universidad Nacional de Colombia. Grupo de Apoyo Pedagógico y Formaciôn Docente, Facultad de Medicina, Universidad Nacional. En: Revista de la Facultad de Medicina; 49 (3):132-140.

RUIZ, Lidia y PACAHANO, Lizabeth (2008). La docencia universitaria y las prácticas evaluativas. Universidad de los Andes. Núcleo Universitario "Rafael Rangel", Edo. Trujillo. Venezuela.

TOCAS $\mathrm{R}_{r}$ Enrique Ramón (2008). Dilemas del docente universitario frente al proceso de evaluación de los aprendizajes. En: Revista Científica THZOECOEN Universidad Senor de Sipan.

UNESCO (1998). Memorias de la Conferencia General de la 29a Reunión de la UNESCO. Paris. En: http://unesdoc.unesco.org/images/0011/001102/110220s. pdf. Información consultada el 15 de Junio de 2012.

(2002). Proyecto Regional de Educación para América Latina y e Caribe (PRELAC), La Habana. En: http://portal.unesco.org/geography/es/ev.php-TOPIC\&URL_SECTION=201.html. Información consultada el 15 de Junio de 2012.

UNIVERSIDAD DE EXTREMADURA (1981). Departamento de Ciencias de la Educación. Paradigmas y modelos de la evaluación educativa. Tomado de Tenbrink.

UNIVERSIDAD SURCOLOMBIANA (2006). Proyecto Educativo Pedagógico. Programa de Pedagogía Inf antil. Neiva.

2005). Consejo Superior Universitario. Acuerdo 048 de 2005.

ZAMBRANO L. Armando (2001). Pedagogía, Educabilidad y Formación de. Docentes. Cali: Nueva Biblioteca Pedagógica. 